удК 621.374

\title{
АНАЛИТИЧЕСКОЕ ОПРЕДЕЛЕНИЕ ПАРАЗИТНЫХ ЧАСТОТ ГЕТЕРОДИННОЙ СИСТЕМЫ, КОТОРЫЕ СОВПАДАЮТ С ТРЕБУЕМОЙ ЧАСТОТОЙ ВЫХОДНОГО СИГНАЛА
}

\author{
Ш. СИНГХ ${ }^{1,2}$, С. Ч. БЕРА ${ }^{1}$, П. П. КУМА ${ }^{1}$, Д. ПЬЮАР ${ }^{2}$ \\ ${ }^{1}$ Индийская организаџия космических исследований, \\ Индия, Ахмадабад \\ ${ }^{2}$ Университет Нирмы, \\ Индия, Ахмадабад, Гуджарат
}

\begin{abstract}
Аннотация. В статье получены формулы для определения самого низкого порядка и других высших порядков паразитных частот, которые совпадают с требуемыми частотами выходного сигнала смесителей. Предложенный подход дает общее выражение, которое используется для любого порядка гетеродинного смешения. Формулы проверены на типичном примере и полученные результаты сравнивались с результатами моделирования, полученными с помощью программы моделирования Advanced Design System. Формулы дают возможность непосредственно получить порядок проблемных паразитных частот, с которыми проектировщики могут столкнуться при разработке гетеродинных систем. В отличие от этих прямых формул, результаты существующих программ для анализа паразитных явлений основаны на максимальном порядке проведенного моделирования. На основе результатов такого моделирования совпадающие паразитные составляющие необходимо сортировать вручную. Предложенные формулы представляют собой экспресс средства для разработчиков систем микроволновой радиосвязи и СВЧ-схем, которые позволяют осуществлять выбор и уточнение частот гетеродинирования в разрабатываемых конструкциях без использования средств моделирования.
\end{abstract}

Ключевые слова: преобразование с понижением частоты; смешение на основной частоте; смешение на гармониках; нелинейный; порядок смешения; паразитный; преобразование с повышением частоты

\section{1. ВВЕДЕНИЕ}

Выбор частоты и проектирование локального генератора колебаний LO (local oscillator) для гетеродинных систем RF (радиочастота) включает исследование паразитных частот, которые возникают при смешении частот внутри системы. Это обусловлено тем, что многие из этих паразитных частот могут совпадать с требуемой частотой выходного сигнала системы. Подобной ситуации нельзя избежать, поскольку генерирование частот произведения присуще всем нелинейным устройствам и вполне очевидно из анализа нелинейной зависимости между током и напряжением, рассмотренной в [1]. Указанную зависимость можно записать в следующем виде:

$$
i=a v+b v^{2}+c v^{3}+\ldots,
$$

где напряжение $v$ соответствует сумме двух входных сигналов, подлежащих смешению. Здесь степень показательной зависимости напряжения $v$ определяется как порядок смешения.

Рассмотрим два синусоидальных входных сигнала с частотами $f_{1}$ и $f_{2}$, которые подаются на нелинейное устройство. Результирующие 


\section{БИБЛИОГРАФИЧЕСКИЙ СПИСОК}

1. Maas, S. A. Nonlinear Microwave and $R F$ Circuits. Norwood, MA: Artech House, 2003.

2. Edwards, M. End-To-End System Design: Advantages of an Integrated Tool For Frequency Planning, Budget Analysis and More. Visual System Simulator, White Paper, 2010. URI: http://www.awrcorp.com/sites/ default/files/content/attachments/VSS-System-PlanningWhite-Paper.pdf.

3. Kirshman, J. Understanding and Correctly Predicting Critical Metrics for Wireless RF Links, Visual
System Simulator, White Paper, 2012. URI: http://www. awrcorp.com/sites/default/files/WP-RFLNK-VSS-2012.1 2.6.pdf.

4. Faria, D.; Dunleavy, L.; and Svensen, T. The Use of Intermodulation Tables for Mixer Simulations. Microwave J. URI: http://www.microwavejournal.com/ articles/3430-the-use-of-intermodulation-tables-for-mixe r-simulations

5. Ismail, A.; Abidi, A. A. A 3.1-to-8.2 GHz zero-IF receiver and direct frequency synthesizer in 0.18-micrometer SiGe BiCMOS for mode-2 MB-OFDM UWB communication. IEEE J. Solid-State Circuits, vol. 40, no. 12, p.2573-2582, 2005. DOI: 10.1109/JSSC. 2005. $\underline{857423}$.

6. Treuttel, J.; Maestrini, A.; Alderman, B.; Wang, H.; Matheson, D.; De Maagt, P. Design of a combined tripler-subharmonic mixer at $330 \mathrm{GHz}$ for multipixel application using European Schottky diodes. 21st ISSTT, Oxford, Apr. 2010. URI: http://www.nrao.edu/meetings/ isstt/papers/2010/2010414417.pdf.

7. Mordachev, V.; Sinkevich, E. Spurious and intermodulation response analysis of passive double-balanced mixers using the double-frequency scanning technique. Proc. of 2013 Int. Symp. on Electromagnetic Compatibility, 2-6 Sept. 2013, Brugge, Belgium. IEEE, 2013. URI: http://ieeexplore.ieee.org/document/66533 97/.

8. Tang, Z.; Pan, S. Image-reject mixer with large suppression of mixing spurs based on a photonic microwave phase shifter. J. Lightwave Technol., vol. 34, no. 20, pp. 4729-4735, 2016. DOI: 10.1109/JLT.2016.2550180.

9. Tang, Z.; Pan, S. A reconfigurable photonic microwave mixer using a $90^{\circ}$ optical hybrid. IEEE Trans. Microwave Theory Tech., vol. 64, no. 9, pp. 3017-3025, 2016. DOI: 10.1109/TMTT.2016.2594278. 